\title{
Non-celiac gluten sensitivity: an emerging syndrome with many unsettled issues
}

\author{
Umberto Volta, Giacomo Caio, Francesco Tovoli, Roberto De Giorgio \\ Department of Medical and Surgical Sciences, Sant'Orsola-Malpighi Hospital, University of Bologna, Bologna, Italy
}

\begin{abstract}
Non-celiac gluten sensitivity is still an undefined syndrome with several unsettled issues despite the increasing awareness of its existence. Gluten is likely responsible for the clinical picture in a subset of patients, whereas in other cases it concurs to this syndrome together with fermentable mono-oligo-disaccharides and polyols and wheat proteins (e.g., amylase trypsin inhibitors). Innate immunity plays a pivotal role in the development of this syndrome, which is characterized by gut inflammation without villous atrophy and likely changes of intestinal barrier function. Data on its epidemiology are still undefined and largely variable. In the USA its prevalence varies from $0.6 \%$ to $6 \%$ in primary or tertiary care, respectively. Clinically, patients complain of gastrointestinal and extra-intestinal symptoms triggered by the ingestion of gluten without evidence of celiac disease and wheat allergy. Intestinal symptoms resemble those of irritable bowel syndrome, whereas neurological signs are quite common among extra-intestinal manifestations. So far, there are no biomarkers for non-celiac gluten sensitivity, but about half of patients shows anti-gliadin antibodies of immunoglobulin G class. Although not specific for non-celiac gluten sensitivity, the detection of such antibodies can support the diagnosis in patients with gluten-related symptoms. In the absence of diagnostic biomarkers a double-blind, placebo-controlled food challenge is currently the best way for confirming non-celiac gluten sensitivity. Studies aimed at clarifying the pathophysiological, clinical and laboratory features of non-celiac gluten sensitivity will help a better management of patients with this novel and intriguing clinical entity.
\end{abstract}

\section{Introduction}

In the last years, clinicians have become progressively aware of a new clinical challenge in the field of gluten-related disorders, i.e. a condition referred to as

Correspondence: Umberto Volta, Laboratory of Immunology, Department of Medical and Surgical Sciences, Sant'OrsolaMalpighi Hospital, University of Bologna, via Massarenti 9, 40138 Bologna, Italy.

Tel.: +39.051.307315 - Fax: +39.051.340877

E-mail: umberto.volta@aosp.bo.it

Key words: non-celiac gluten sensitivity, innate immunity, epithelial barrier function, amylase trypsin inhibitors, fermentable oligo-, di-, and monosaccharides, and polyols, anti gliadin antibodies.

Contributions: UV and RDG conceived and wrote the review; GC revised and implemented parts of the manuscript with experimental data; FT helped with references and editing.

Conflict of interests: the authors declare no conflict of interests.

Received for publication: 28 November 2013.

Accepted for publication: 2 December 2013.

This work is licensed under a Creative Commons Attribution NonCommercial 3.0 License (CC BY-NC 3.0).

(C) Copyright U. Volta et al., 2014

Licensee PAGEPress, Italy

Italian Journal of Medicine 2014; 8:225-231

doi:10.4081/itjm.2013.461 non-celiac gluten sensitivity (NCGS). Patients with such syndrome complain of symptoms related to gluten ingestion in the absence of celiac disease (CD) and wheat allergy (WA). ${ }^{1,2}$ The reasons to explain the occurrence of NCGS in the clinical setting are still undefined. However, a number of factors are thought to have exerted a role over the years. First, the mechanization of farming and the growing industrial use of pesticides certainly played a role in selecting variants of wheat containing high amount of toxic gluten peptides. This has clearly contributed to increase the spread of gluten-related symptoms in the general population. ${ }^{3,4}$ Secondly, another possible factor should be ascribed to the reduced time of dough fermentation, resulting in a higher content of toxic gluten fractions in bread and bakery products compared to the past. Thirdly, the worldwide spread of the Mediterranean diet based on a high intake of gluten-containing foods may play a role in triggering gluten-related symptoms. Finally, the media hype has drawn the attention of people towards gluten as a potential toxic element, claiming that a gluten free diet represents healthy food style. $^{5}$ Thus, media hype led many subjects to believe that their symptoms were due to gluten. Nonetheless, a clear-cut correlation between the ingestion of gluten and appearance of symptoms in a proportion of these patients has strengthened the awareness of a new gluten-related disorder distinct from CD and WA in the scientific community. ${ }^{6,7}$

From a clinical stand point, NCGS is characterized 
by a wide array of gastro-intestinal and extra-intestinal symptoms, occurring shortly after gluten ingestion, improving (or even disappearing) in a few days when gluten is withdrawn from the diet and recurring rapidly when gluten is reintroduced. ${ }^{2,6,7}$ A mandatory prerequisite for suspecting this condition is to rule out the diagnosis of both $\mathrm{CD}$ and WA when patients are on a gluten-containing diet. Although NCGS has been only recently recognized as a new gluten related syndrome, its existence has been postulated more than 30 years ago by means of a double-blind cross-over performed in 8 non-celiac women with diarrhea and abdominal pain, confirming that the symptoms were elicited by gluten in 6 of them. ${ }^{8}$ In 2000, a Finnish group demonstrated that a large proportion of patients with positivity for anti-gliadin antibodies and normal intestinal mucosa complained of intestinal symptoms induced by gluten ingestion, therefore extending the concept of gluten sensitivity beyond the well-recognized CD. ${ }^{9}$ It is unclear why these two papers did not reach the awareness of the scientific community. However, this behavior certainly contributed to relegate NCGS in the no man's land for many years. ${ }^{10}$

Over the last 3 years the experts of gluten related disorders have started again to work on this condition as shown by the two Consensus Conferences, held in London (2011) and Munich (2012), and by several scientific original papers and reviews on this topic. ${ }^{1,2,6,7,11-14}$ Although there is a general agreement that NCGS does exist, our knowledge on this syndrome is still lacking with many aspects waiting to be clarified. Scarce information has been generated on its pathogenesis, epidemiology and diagnostic biomarkers. Moreover, whether NCGS is a transient or a permanent disorder still remains an open issue. One of the most critical aspects is the absence of specific biomarkers which does not allow to identify with certainty NCGS. This condition is therefore diagnosed only clinically based on symptom evaluation following gluten ingestion.

This review has been designed to provide a practical guidance to general practitioners and internists as to pathophysiology, diagnosis and management of patients with NCGS.

\section{Pathogenesis}

The pathogenic mechanisms of NCGS are far to be established. Preliminary data suggest that NCGS is an immune mediated disorder, triggered by an activation of innate immunity without a well-demonstrated involvement of adaptive immunity which is instead a pivotal factor in the development of $\mathrm{CD} .{ }^{15-17}$ The activation of innate immunity in NCGS is supported by the increased expression of toll-like-receptors (TLRs) in the small intestine. Particularly, TLR2 and, to a lesser extent, TLR1 and TLR4, a class of proteins that plays a key role in innate immunity, have been identified in the intestinal mucosa and some cells of the lamina propria of patients with NCGS. ${ }^{15}$ At variance with $\mathrm{CD}$, adaptive immunity markers, such as interleukin (IL)17A, IL-6, IFN- $\gamma$, IL-17 and IL-21, have not been observed to be over-expressed in the duodenal mucosa of NCGS patients. ${ }^{16}$ Furthermore, NCGS differs from $\mathrm{CD}$ in terms of duodenal expression of $\mathrm{T}_{\mathrm{REG}}$ marker FOXP3 which is reduced in gluten sensitive patients compared to celiacs. Therefore, the reduced expression of FOXP3 might be interpreted as a result of a less prominent activation of adaptive immunity in NCGS vs CD.

The epithelial barrier function of the intestinal mucosa has been investigated in NCGS. ${ }^{1,15}$ Differently from CD, NCGS patients usually show a reduced intestinal permeability measured by a lactulose/mannitol absorption test, suggesting an increased intestinal barrier function in this condition. ${ }^{15} \mathrm{~A}$ significantly higher expression of claudin-4 mRNA, a marker of reduced permeability, has been found in duodenal biopsies of NCGS patients. Different results have been reported by Vazquez-Roque et al. who found an increased intestinal permeability in a subgroup of human leukocyte antigen (HLA)-DQ2/DQ8+ patients with diarrhea-predominant irritable bowel syndrome following gluten challenge. ${ }^{18}$ This discrepancy implies that further studies are necessary to define small intestinal permeability in NCGS.

Experimental studies have shown that HLA-DQ8 transgenic mice sensitized by gliadin have an increased secretion of acetylcholine from the myenteric plexus and this results in an enhanced muscle contractility and epithelial hypersecretion. Both abnormalities disappeared after gluten withdrawal. ${ }^{19}$ Moreover, changes of the intestinal microbiota with an increased concentration of Gram bacteria and a decreased number of $\mathrm{Gram}^{+}$Lactobacilli might favour the inflammatory response to gluten. ${ }^{20}$

Recent evidence indicated that gluten proteins are not the only factor responsible for the clinical picture of NCGS. Indeed, other wheat proteins are likely to play a role in determining this syndrome in most patients. In particular, the attention of researchers has been focused on wheat amylase trypsin inhibitors (ATIs), which are strong activators of innate immune responses in monocytes, macrophages and dendritic cells. ${ }^{21}$ ATIs might trigger inflammatory and immune responses in a variety of disorders including NCGS, $\mathrm{CD}$ and Baker's asthma.

A diet rich in fermentable oligo-, di-, and monosaccharides and polyols (FODMAPs) can elicit the clinical picture of NCGS. ${ }^{22}$ FODMAPs are poorly absorbed short-chain carbohydrates, that, due to their small molecular size and rapid fermentability, cause 
the distension of the intestinal lumen with liquid and gas leading to functional gastrointestinal symptoms. Common food sources of FODMAPs are grains and cereals (wheat, rye, barley), milk, legumes, honey, fruits (watermelon, cherry, mango, pear) and vegetables (chicory, fennel, beetroot, leek). A low FODMAPs diet significantly improves the functional gastrointestinal symptoms in NCGS patients. ${ }^{22}$ Functional gastrointestinal symptoms observed in patients with NCGS as well as with other disorders including irritable bowel syndrome (IBS) could be also partly related to food additives such as glutamates, benzoates, sulphites and nitrates which are added to many commercial products for different reasons (in order to improve flavor, color and preservative function).

\section{Epidemiology and clinical picture}

The prevalence of NCGS is still undefined and the few data available are largely variable according to primary care or tertiary referral centres in the United States. Data from the National Health and Nutrition Examination Survey (NHANES), a primary care program based on interviews, physical examination and blood samples, showed 49 cases of suspected NCGS over 7762 patients $(0.6 \%)$ (age range 6-80 years) in the 2009-2010 period. ${ }^{23}$ On the other hand, in the tertiary care Center for Celiac Research, University of Maryland, NCGS was recognized in 347 over 5896 patients in the 2004-2009 period with a prevalence of $6 \% .^{2}$ Although it is expected that the majority of NCGS patients are seen at specialist centers, this cannot explain the huge difference found in these two studies. A recent paper has underlined the close linkage between NCGS and IBS, showing that $30 \%$ of 920 patients with IBS had NCGS associated with multiple food hypersensitivity in the majority of cases. ${ }^{24}$ Since it is well established that IBS is a very common condition found in $16 \%-25 \%$ of the general population, ${ }^{25,26}$ it is very much likely that also NCGS is characterized by a high prevalence in the general population. Over the last 12 months (from November 1, 2012 to October 31, 2013), 2340 subjects were seen at the outpatient clinics of the Department of Medical and Surgical Sciences of the University of Bologna. The criteria for NCGS were fulfilled by 84 (1:28; $3.5 \%$ ) patients (Table 1). Of these, 66 were females and 18 males (F/M: 3.7:1), with a mean age of 38 years (range 16-65). In the same period the diagnosis of $\mathrm{CD}$ was performed in 48 (mean age 36 years, range 17-64) out of the 2340 patients seen $(1: 49 ; 2.1 \%)$. The ratio between NCGS/CD was 1.75:1. Based on our 1year experience, the expected prevalence of NCGS in the general population might be slightly higher than that of $\mathrm{CD}$ which is $1 \%$ worldwide.

Because of its clinical picture NCGS can be regarded as a syndrome rather than a unique disorder, being characterized by a variety of gluten-related gastrointestinal and extra-intestinal symptoms occurring in patients in whom the diagnosis of CD and WA had been previously excluded.

The interval time between gluten ingestion and the appearance of symptoms in NCGS varies from some hours and a few days and it is intermediate between that of wheat allergy and CD. Indeed, in wheat allergy symptoms usually appear in a few minutes after gluten exposure, whereas in celiac disease the interval time

Table 1. Symptoms prevalence of patients with non-celiac gluten sensitivity observed at the Department of Medical and Surgical Sciences of the University of Bologna over the last 12 months.

\begin{tabular}{llll}
\hline \multicolumn{4}{c}{ NCGS patients 84/2340 (3.5\%); mean age 38 years (range 16-65 years); F/M: 3.7:1* } \\
\hline Gastrointestinal symptoms & $\mathbf{\%}$ & Extra-intestinal symptoms & \% \\
\hline Abdominal pain & 84 & Tiredness & 78 \\
\hline Bloating & 84 & Headache & 55 \\
\hline Diarrhea & 55 & Foggy mind & 40 \\
\hline Nausea or vomiting & 50 & Anxiety & 38 \\
\hline Gastric pain & 48 & Joint/muscle pain fibromyalgia-like & 30 \\
\hline Heartburn & 45 & Numbness (arms, legs, fingers) & 30 \\
\hline Aerophagia & 35 & Skin rash/dermatitis & 27 \\
\hline Aphthous stomatitis & 30 & Anemia & 22 \\
\hline Gastro-esophageal reflux disease & 30 & Weight loss & 10 \\
\hline Altered bowel habits & 27 & Rhinitis & 9 \\
\hline Constipation & 24 & Asthma & 5 \\
\hline
\end{tabular}

NCGS, non-celiac gluten sensitivity. *The majority of patients displayed more than 2 gastrointestinal and extra-intestinal symptoms. 
between gluten ingestion and the clinical manifestation can be very long (up to weeks or years). ${ }^{1,17,27}$ In a previous work from our group the clinical and serological features of NCGS were investigated in seventy-eight non-consecutive patients (56 females e 22 males, median age 38 years, range 17-63), diagnosed in our center between January 2009 and June 2011. ${ }^{28}$ In all of them NCGS was suspected on the basis of symptoms elicited by gluten ingestion after the exclusion of CD by both serology [negativity for anti endomysial (EmA) and tissue transglutaminase antibodies (tTGA)] and duodenal biopsy (absence of villous atrophy) and WA by specific immunoglobulin (Ig)E/prick tests to wheat. NCGS diagnosis was confirmed by a trial of gluten free diet (GFD) for 6 months with a quick disappearance of symptoms, followed by an open gluten challenge of 1 month with an immediate relapse of the clinical picture. All NCGS patients showed both gastrointestinal and extra-intestinal symptoms occurring in a few hours or days after gluten ingestion. Among gastrointestinal symptoms the most frequent manifestations were abdominal pain and bloating, followed by diarrhea and, less frequently, constipation. Among extra-intestinal signs the most frequent symptom was mental confusion or foggy mind, defined as sensation of lethargy elicited by gluten, followed by fatigue, skin rash, headache, joint and muscle pain (fibromyalgia-like syndrome), leg or arm numbness, depression and anxiety and anemia. Clinical features of NCGS emerged from this study showed that this syndrome is more frequent in middle-aged adults, being found also in adolescents and in old people. Moreover, similarly to celiac disease NCGS resulted to be much more frequent in females than in males. NCGS has been rarely found in children. In a recent paper it has been reported the first series of 15 children with NCGS. ${ }^{29}$ In these young patients ( 10 males and 5 females, mean age 9 years) gastrointestinal symptoms (abdominal pain $80 \%$, diarrhea $73 \%$, bloating $26 \%$ ) were more frequent than extraintestinal signs (mainly tiredness and headache, found in $33 \%$ and $20 \%$ of cases, respectively).

Among the several unsettled issues of NCGS it remains still unclear whether NCGS patients are more prone to autoimmune disorders and complications like to $\mathrm{CD}$ patients. A few published studies suggest that the finding of autoimmune disorders in NCGS would be a rare event. ${ }^{2,28}$ Indeed, in one of our previous studies none of the 78 NCGS patients displayed type 1 diabetes mellitus and only one (1.3\%) had autoimmune thyroiditis, compared with $5 \%$ and $19 \%$, respectively, of 80 patients with $\mathrm{CD} \cdot{ }^{28}$ Another relevant point that needs to be explored is whether patients with NCGS can develop well-known CD-associated complications, such as ulcerative jejuno-ileitis, collagenous sprue, small intestinal lymphoma and other gastroin- testinal neoplasms. ${ }^{2,17}$ Since NCGS has been only recently identified, its follow-up is still too short to have solid information on the outcome of this condition.

\section{Diagnostic criteria}

So far the diagnosis of NCGS relies on the accurate assessment of the clinical features along with the exclusion of WA and $\mathrm{CD}$, according to the diagnostic criteria suggested by the Consensus Conference on NCGS published by Sapone et al. ${ }^{2}$ These criteria establish that the exclusion of gluten from the diet is followed by a significant improvement up to the disappearance of intestinal and extra-intestinal symptoms, while gluten reintroduction evokes symptoms. Since it is impossible to exclude that the improvement of the clinical picture after GFD can be induced by a placebo effect, a double-blind, placebo-controlled challenge (DBPC) is strongly recommended to validate the diagnosis of NCGS. From a practical point of view this procedure is complex and not easy to perform for diagnostic purpose. Up-to-now these trials have been performed only in patients with IBS, with NCGS manifesting discordant results. ${ }^{24,30,31}$ Two of these studies, performed by the same group, firstly confirmed the existence of NCGS showing the significant recurrence of the clinical manifestations in patients ingesting gluten compared to the placebo group. ${ }^{30}$ In a second DBPC the same group concluded that gluten elicited neurological, but not intestinal symptoms in a few patients with IBS, whereas in the majority of them, symptoms were attributable to FODMAPs. ${ }^{31}$ In support of this latter possibility, a low FODMAPs diet determined a significant improvement of symptoms in the same patients. Similar results to the first study of Biesiekierski et al. were obtained by Carroccio et al. whose DBPC confirmed a significant worsening of intestinal and extra-intestinal symptoms in the gluten $v s$ the placebo group. ${ }^{24}$

Before suspecting NCGS both WA and CD must be excluded by appropriate tests performed on a gluten containing diet. WA should be ruled out by testing for serum IgE antibodies to gluten proteins and wheat fractions as well as skin Prick tests, whereas CD must be excluded by the negativity of specific serological tests, such as IgA tTGA, IgA EmA and IgG deamidated gliadin antibodies and by the absence of villous atrophy in the small intestinal biopsy. ${ }^{1}$

The lack of a specific biomarker hampers the reliability of NCGS diagnosis. The only serological marker found in a proportion of patients with NCGS is the first generation antibody to gliadin (AGA).,24,28 AGA positivity has been found in the sera of about the half of NCGS patients and it is almost always confined to IgG class (only occasionally belonging to the IgA class). ${ }^{28}$ AGA is not a specific test for NCGS being 
positive in many other conditions such as autoimmune liver diseases, irritable bowel syndrome, connective tissue disorders and even blood donors, but, for the time being, its positivity (especially at a high titer) in patients with suspected NCGS can contribute to this diagnosis. In contrast to $\mathrm{CD}$, AGA disappear in almost all patients with NCGS within 6 months of GFD (Volta et al., 2013, unpublished data). It is reasonable to hypothesize that an immunological memory might be active in CD but not in NCGS. ${ }^{32}$

According to the Consensus Conference criteria, ${ }^{2,7}$ a duodenal biopsy is highly recommended in patients with suspected NCGS when they are on a gluten containing diet in order to rule out $\mathrm{CD}$ diagnosis even if the celiac serology is negative (possibility of seronegative $\mathrm{CD}$ which occurs in $1-2 \%$ of total CD cases). ${ }^{17}$ The majority of NCGS patients display a normal histology of duodenal mucosa with a number of intraepithelial lymphocytes (IELs) within 25 per 100 epithelial cells (grade 0 according to Marsh-Oberhüber modified classification), ${ }^{33}$ whereas a lower number of cases show a slight increase of IEL ranging from 25 to 40 per 100 epithelial cells consistent with a low grade of inflammation. ${ }^{28}$ At variance with $\mathrm{CD}$, in NCGS there is no increase of T-cell receptor $\gamma / \delta$ IELs. ${ }^{15}$

NCGS does not show any correlation with the HLA. Positivity for HLA-DQ2 and/or-DQ8 in NCGS patients is very close to that detected in the general population and far lower than that found in CD patients. ${ }^{2,28}$ Serological, genetic and histopathological features of NCGS cases diagnosed in our center over the last 12 months are reported in Table 2.

A prospective survey on NCGS showed that in a not negligible number of cases (up to 20\%) laboratory

Table 2. Serology, genetics and duodenal histology in non-celiac gluten sensitivity cases observed at the Department of Medical and Surgical Sciences of the University of Bologna over the last 12 months.

NCGS patients 84/2340 (3.5\%);

mean age 38 years (range 16-65 years); F/M: 3.7:1*

\begin{tabular}{lc}
\hline Analyses & $\mathbf{\%}$ \\
\hline Serology & \\
AGA IgG & 48 \\
AGA IgA & 7 \\
DGP-AGA IgG & 2 \\
\hline Genetics* & \\
HLA-DQ2 & 35 \\
HLA-DQ8 & 5
\end{tabular}

\section{Duodenal biopsy ${ }^{\circ}$}

Marsh-Oberhüber 0

Marsh-Oberhüber 1

35

NCGS, non-celiac gluten sensitivity; AGA, anti-gliadin antibodies; IgG, IgA, immunoglobulins $\mathrm{G}$ and A; DGP, deamidated gliadin antibodies; HLA, human leukocyte antigen. *Performed only in 20 cases; ${ }^{\circ}$ duodenal biopsy performed in 64 cases. tests can disclose signs of malabsorption very similar to those observed in $\mathrm{CD}$, such as low levels of ferritin, folic acid and vitamin D and B12. ${ }^{34}$ Bone densitometry can show evidence of osteopenia, particularly in patients with low levels of vitamin $\mathrm{D}$ and calcium associated with increased parathormone levels. In some cases NCGS patients showing absorption abnormalities have significant improvement of biochemical parameters after GFD.

\section{Treatment}

There are no established guidelines regarding treatment of patients with NCGS. First of all, it is highly recommendable that people should not eliminate gluten from diet without a specific indication given by their physicians; also, auto-diagnosis of a presumed sensitivity to dietary gluten should be avoided. Moreover, gluten removal is absolutely useless in cases lacking a substantiated diagnosis of NCGS. On the other hand, a diagnosis of NCGS should be established in a referral center following the previously mentioned work-up. In case of gluten sensitivity, patients will be advised to change their dietary habits and start to consume gluten-free foods. Cereals such as rice, corn, buckwheat, millet and leguminosae, e.g. quinoa, amaranth and soybean, are recommended as substitutes of gluten-containing products. ${ }^{35}$ Commercially available gluten-free products are useful for patients with NCGS to achieve a thoroughly glutenfree regimen, but, as also recommended for $\mathrm{CD}$ patients, their consumption should be integrated with the ingestion of meat, fish, eggs, fruit and vegetables which are naturally gluten-free foods. In thorough GFD regimen, the consumption of commercially available gluten-free products should be not so high in order to limit the introduction of chemical additives and preservatives highly represented in these products and potential causes of functional gastrointestinal symptoms. ${ }^{6,22}$ Currently, it is unknown whether NCGS patients should care about gluten contamination inadvertently introduced by foods as for CD patients. Moreover, it should be underlined that the level of tolerance varies in the single individual and there are also subjects with NCGS who do not tolerate even small amounts of gluten. GFD leads to the complete disappearance of symptoms in a proportion of patients with NCGS, whereas in other cases the improvement after GFD is only partial. In these cases a low FODMAPs diet in addition to gluten withdrawal can significantly improve the clinical picture. It is still unclear whether NCGS is a permanent or transient condition. ${ }^{1,2,7}$ A few patients with NCGS reintroduce gluten in their diet after the significant improvement achieved by means of GFD because they fear to experience again the wide array of gluten-induced symptoms. Theoretically, the reintroduction of gluten after 1-2 years of GFD might be advised. A correct approach might be a desen- 
sitization trial by introducing progressively small amounts of gluten.

\section{Conclusions}

Although NCGS has been recognized as a new gluten related syndrome from the scientific community, our knowledge on this condition is still in progress and there are many unsettled issues that need clarification. ${ }^{36}$ Innate immunity seems to be the main trigger of NCGS, but further studies are awaited to explore the possible role of adaptive immunity. ${ }^{37,38} \mathrm{~A}$ low grade of inflammation in intestinal mucosa with intestinal permeability changes and alterations of intestinal microbiota are likely relevant in the development of this syndrome, but their role is far to be established. ${ }^{1,2,6,7}$ A close link between gluten and symptom generation has been clearly demonstrated in NCGS patients, but other proteins contained in wheat have the potential to cause symptoms experienced by NCGS patients. In this context, wheat ATIs, which are proteins highly resistant to intestinal proteases and elicit innate immunity, could trigger NCGS. ${ }^{21}$ It has been demonstrated that a subgroup of patients with NCGS recognizes gluten as the only cause of symptoms, but in other NCGS patients a multiple food hypersensitivity underlies the clinical picture. ${ }^{39}$ In this context, it has been shown that a diet rich in FODMAPs, present not only in gluten-containing ce-

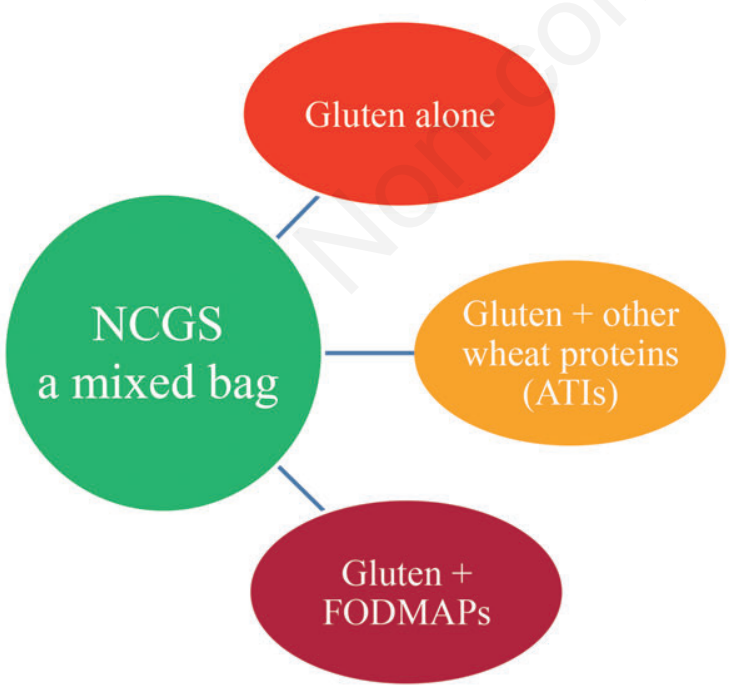

Figure 1. Non-celiac gluten sensitivity (NCGS) can be regarded as a mixed bag with different triggers generating the clinical picture. In some patients gluten causes symptoms by itself alone, while in many others it concurs to the clinical picture together with wheat amylase-trypsin inhibitors (ATIs), and fermentable oligo-, di-, and monosaccharides and polyols (FODMAPs). reals, but also in milk, honey and legumes might trigger NCGS. ${ }^{31}$ Overall, gluten sensitivity can be viewed as a mixed bag with many triggers (gluten, ATIs, FODMAPs) evoking the clinical picture (Figure 1). Furthermore, chemical additives, such as glutamates, benzoates, sulphites and nitrates, which are added to many commercial products might have a role in evoking functional gastrointestinal symptoms of NCGS and other disorders characterized by a mild intestinal inflammation such as IBS. ${ }^{6,22}$ In this respect, a subgroup of NCGS patients are not expected to improve by eating commercially available gluten-free products (as they are usually rich of additives and preservatives), rather they improve with a diet based on natural gluten-free foods. ${ }^{6}$

The different combination of genetic, histological and serological features observed in NCGS patients confirms the heterogeneity of this syndrome. Some patients with NCGS display positivity for HLA-DQ2 and/or -DQ8 and/or positivity for AGA and/or mild intestinal damage (Marsh 1 lesion), which are non-diagnostic for $\mathrm{CD}$, but might eventually progress towards a florid CD. Although it seems likely that NCGS and CD are two separate entities the possibility that NCGS may evolve to CD cannot be disregarded. The detection of IgA deposits in duodenal biopsies might help to identify among NCGS those who are at risk of developing $\mathrm{CD} .{ }^{40}$

The natural history of NCGS is still too short to establish whether this syndrome can be associated with development of autoimmune disorders and can display complications (ulcerative jejunoileitis intestinal lymphoma and small bowel carcinoma) in a similar fashion to CD. It is still unclear whether NCGS is a transient or a permanent condition, but it is tempting to speculate a sort of desensitization by reintroducing small amounts of gluten in these patients after some years of GFD.

In conclusion, further studies are eagerly awaited to shed light on the sill many unsettled aspects inherent to NCGS, a new complex clinical challenge amongst gluten-related disorders.

\section{References}

1. Volta U, De Giorgio R. New understanding of gluten sensitivity. Nat Rev Gastroenterol Hepatol 2012;9:295-9.

2. Sapone A, Bai JC, Ciacci C, et al. Spectrum of glutenrelated disorders: consensus on new nomenclature and classification. BMC Medicine 2012;10:13.

3. Belderok B. Developments in bread-making processes. Plant Food Hum Nutr 2000;55:1-86.

4. Blomfeldt TO, Kuktaite R, Johansson E, Hedenqvist MS. Mechanical properties and network structure of wheat gluten forms. Biomacromolecules 2011;12:1707-15.

5. Di Sabatino A, Corazza GR. Non-celiac gluten sensitivity: sense or sensibility? Ann Intern Med 2012;156:309-11. 
6. Volta U, Caio G, Tovoli F, De Giorgio R. Non-celiac gluten sensitivity: questions still to be answered despite increasing awareness. Cell Mol Immunol 2013;10:383-92.

7. Catassi C, Bai JC, Bonaz B, et al. Non-celiac gluten sensitivity: the new frontier of gluten related disorders. Nutrients 2013;5:3839-53.

8. Cooper BT, Holmes GK, Ferguson R, et al. Gluten-sensitive diarrhea without evidence of celiac disease. Gastroenterology 1980;79:801-6.

9. Kaukinen K, Turjanmaa K, Mäki M, et al. Intolerance to cereals is not specific for celiac disease. Scand J Gastroenterol 2000;35:942-6.

10. Verdu EF, Armstrong D, Murray JA. Between celiac disease and irritable bowel syndrome: the 'no man's land' of gluten sensitivity. Am J Gastroenterol 2009;104:1587-94.

11. Troncone R, Jabri B. Coeliac disease and gluten sensitivity. J Intern Med 2011;269:582-90.

12. Lundin KEA, Alaedini A. Non-celiac gluten sensitivity. Gastrointest Endosc Clin N Am 2012;22:723-34.

13. Brown AC. Gluten sensitivity: problems of an emerging condition separate from celiac disease. Expert Rev Gastroenterol Hepatol 2012;6:41-53.

14. Rostami K, Hogg-Kollars S. Non-coeliac gluten sensitivity: a patient's journey. BMJ 2012;345:e7982.

15. Sapone A, Lammers K, Casolaro V, et al. Divergence of gut permeability and mucosal immune gene expression in two gluten-associated conditions: celiac disease and gluten sensitivity. BMC Medicine 2011;9:23.

16. Sapone A, Lammers KM, Mazzarella G, et al. Differential mucosal IL-17 expression in two gliadin-induced disorders: gluten sensitivity and the autoimmune enteropathy celiac disease. Int Arch Allergy Immunol 2010;152:75-80.

17. Volta U, Villanacci V. Celiac disease: diagnostic criteria in progress. Cell Mol Immunol 2011;8:96-102.

18. Vazquez-Roque MI, Camilleri M, Smyrk T, et al. A controlled trial of gluten-free diet in patients with irritable bowel syndrome-diarrhea: effects on bowel frequency and intestinal function. Gastroenterology 2013;144:903-11.

19. Verdu EF, Huang X, Natividad J, et al. Gliadin-dependent neuromuscular and epithelial secretory responses in gluten-sensitive HLA-DQ8 transgenic mice. Am J Physiol Gastrointest Liver Physiol 2008;294:G217-25.

20. Natividad JM, Huang X, Slack E, et al. Host responses to intestinal microbial antigens in gluten sensitive mice. PLos One 2009;4:e6472.

21. Junker Y, Zeissig S, Kim SJ, et al. Wheat amylase trypsin inhibitors drive intestinal inflammation via activation of toll-like receptor 4. J Exp Med 2012;209:2395-408.

22. Gibson PR, Sheperd SJ. Food choice as a key management strategy for functional gastrointestinal symptoms. Am J Gastroenterol 2012;107:657-66.

23. DiGiacomo DV, Tennyson CA, Green PH, Demmer RT. Prevalence of gluten-free diet adherence among individuals without celiac disease in the USA: results from the Continuous National Health and Nutrition Examination Survey 2009-2010. Scand J Gastroenterol 2013;48:921-5.

24. Carroccio A, Mansueto P, Iacono G, et al. Non-celiac wheat sensitivity diagnosed by double-blind placebo- controlled challenge: exploring a new clinical entity. Am J Gastroenterol 2012;107:1898-906.

25. Breckan RK, Asfeldt AM, Straume B, et al. Prevalence, comorbidity, and risk factors for functional bowel symptoms: a population-based survey in Northern Norway. Scand J Gastroenterol 2012;47:1274-82.

26. Krogsgaard LR, Engsbro AL, Bytzer P. The epidemiology of irritable bowel syndrome in Denmark. A population-based survey in adults $\leq 50$ years of age. Scand J Gastroenterol 2013;48:523-9.

27. Inonata N. Wheat allergy. Cur Opin Aller Clin Immunol 2009;9:238-43.

28. Volta U, Tovoli F, Cicola R, et al. Serological tests in gluten sensitivity (nonceliac gluten intolerance). J Clin Gastroenterol 2012;46:680-85.

29. Francavilla R, Cristofori F, Castellaneta S, et al. Clinical, serologic, and histologic features of gluten sensitivity in children. J Pediatr 2014;164:463-7.e1.

30. Biesiekierski JR, Newnham ED, Irving PM, et al. Gluten causes gastrointestinal symptoms in subjects without celiac disease: a double-blind randomized placebo-controlled trial. Am J Gastroenterol 2011;106:508-14.

31. Biesiekierski JR, Peters SL, Newnham ED, et al. No effects of gluten in patients with self-reported non-celiac gluten sensitivity after dietary reduction of fermentable, poorly absorbed, short-chain carbohydrates. Gastroenterology 2013;145:320-8.

32. Volta U, Corazza GR, Frisoni M, et al. IgA antigliadin antibodies and persistence of jejunal lesions in adult celiac disease. Digestion 1990;47:111-4.

33. Oberhüber G, Granditsch G, Vogelsang H. The histopathology of coeliac disease: time for a stndardized report scheme for pathologists. Eur J Gastroenterol Hepatol 1999;11:1185-94.

34. Volta U. Indagine su sensibilità al glutine non celiaca: risultati preliminari. Celiachia Notizie 2013;1:58-60.

35. Gibert A, Espadaler M, Angel Canela M, et al. Consumption of gluten-free products: should be the threshold value for traces amounts of gluten be at 20, 100 or 200 p.p.m.? Eur J Gastroenterol Hepatol 2006;18:1187-95.

36. Di Sabatino A, Giuffrida P, Corazza GR. Still waiting for a definition of nonceliac gluten sensitivity. J Clin Gastroenterol 2013;47:567-9.

37. Brottveit M, Beitnes AC, Tollefsen S, et al. Mucosal cytokine response after short-term gluten challenge in celiac disease and non-celiac gluten sensitivity. Am J Gastroenterol 2013;108:842-50.

38. Sollid LM, Jabri B. Triggers and drivers of autoimmunity: lessons from celiac disease. Nat Rev Immunol 2013;13:294-302.

39. Carroccio A, Mansueto P, D'Alcamo A, Iacono G. Non celiac wheat sensitivity as an allergic condition: personal experience and narrative review. Am J Gastroenterol 2013;108:1845-52.

40. Not T, Ziberna F, Vatta S, et al. Cryptic genetic gluten intolerance revealed by intestinal antitransglutaminase antibodies and response to gluten-free diet. Gut 2011;60: 1487-93. 